\title{
EDUCAÇÃO PERMANENTE EM SAÚDE: Reflexões e desafios
}

\section{CONTINUING EDUCATION IN HEALTH: Reflections and challenges}

\author{
EDUCACIÓN PERMANENTE EN SALUD: Reflexiones y desafíos
}

\author{
Eliane Marina Palhares Guimarães ${ }^{*}$ \\ SANDRA HaUeisen Martin ${ }^{*}$ \\ Flávia Cristina Paolinelli Rabelo ${ }^{* * *}$
}

\begin{abstract}
RESUMO
Este trabalho é resultado de uma reflexão sobre o processo de educação permanente em saúde e na enfermagem. Tem como objetivo apresentar algumas perspectivas e tendências da educação em saúde e na enfermagem, com ênfase em educação permanente. Analisa a importância dos avanços tecnológicos e sua incorporação no processo de ensinar e de aprender. $\mathrm{O}$ texto é dividido em três partes, abordando inicialmente as mudanças organizacionais e, conseqüentemente, na educação, decorrentes da incorporação tecnológica. Destaca a necessidade de adoção de novos modelos pedagógicos no intuito de (re)construir o conhecimento. Em seguida, discute a educação em enfermagem fazendo uma descrição da situação atual e aponta algumas perspectivas para a capacitação profissional em serviço, entendida como uma decisão política e institucional, na busca de novos modos de ensinar, aprender, assistir e cuidar. Finaliza com uma consideração sobre educação permanente em saúde e na enfermagem, pontuando alguns novos desafios. Esta reflexão permite reafirmar a necessidade de atualização dos profissionais no que diz respeito à utilização de tecnologias que possam responder à demanda social de acesso e alcance das oportunidades de capacitação desenvolvidas nos locais de trabalho.
\end{abstract}

Palavras chave: Educação em saúde, educação em enfermagem, capacitação em serviço.

\begin{abstract}
This work is the result of a reflection on the process of continuing education in health and nursing. It has the objective of presenting some perspectives and tendencies on education in health and nursing, with emphasis in continuing education. It analyzes the importance of technology and its use in the process of teaching and learning. The text is divided into three parts. At first it approaches organizational and educational changes due to the use of technology. It also gives emphasis to the need of the application of new pedagogical approaches with the intention of (re)building knowledge. After that, it highlights education in nursing by giving a description of the current situation and it points out some perspectives related to professional qualification in service taken as an institutional and political decision in the search of new ways to teach, to learn, to attend and to take care. It is concluded with considerations on continuing education in health and nursing pointing out some new challenges. This reflection reaffirms the need of updating the professional workers in the use of technology that can respond to the demand of access and the given conditions to the continuing education at the working environment.
\end{abstract}

Key words: Health education, nursing education, inservice training.

\footnotetext{
“Enfermeira. Docente da Escola de Enfermagem da Universidade Federal de Minas Gerais. Belo Horizonte. Brasil. elianemg@enf.ufmg.br "Enfermeira. Enfermeira Assistencial do CTI Pediátrico e Neonatal do Hospital IPSEMG. Belo Horizonte. Brasil. sandrahmartin@yahoo. com.br

${ }^{* * *}$ Enfermeira do Hospital Regional de Betim. Belo Horizonte. Brasil. flaviapaolinelli@yahoo.com.br.
} 


\section{RESUMEN}

Este trabajo es el resultado de una reflexión en el proceso de la educación permanente en salud y enfermería. Tiene como objetivo presentar algunas perspectivas y tendencias en la educación en salud y enfermería, con énfasis en la educación permanente. Analiza la importancia de los avances tecnológicos y de su incorporación en el proceso enseñanza-aprendizaje. El texto se divide en tres partes, acercando inicialmente los cambios en las organizaciones y en la educación, derivados de la incorporación tecnológica. Separa la necesidad de la adopción de nuevos modelos pedagógicos con la intención de (re)construir el conocimiento. Después, discute la educación en enfermería que hace una descripción de la situación actual y señala algunas perspectivas con respecto a la calificación profesional en servicio, entendida como una decisión política institucional, en la búsqueda de nuevas maneras de enseñar, de aprender, de atender y de tomar a cuidado de. Concluye con una consideración en la educación permanente en la salud y en enfermería y separa algunos nuevos desafíos. Esta reflexión permite reafirmar la necesidad de la actualización de los profesionales respecto al uso de las tecnologías que pueden responder a la demanda social del acceso y al alcance de las ocasiones desarrolladas de la calificación en los sitios de trabajo.

Palabras clave: Educación en salud, educación en enfermería, capacitación en servicio.

Fecha recepción:12/01/08 Fecha aceptación:12/07/10

\section{INTRODUÇÃO}

A educação consome cada vez mais espaço na vida das pessoas em um mundo onde a rapidez das mudanças se conjuga com o fenômeno da globalização, possibilitando aumentar conseqüentemente o nível de participação dos sujeitos nas sociedades modernas. A evolução rápida do mundo exige contínua atualização dos saberes. Desta maneira, a educação age como mola mestre para transformação dos paradigmas dos indivíduos, possibilitando que este compreenda o que ocorre na sociedade, ampliando assim, a visão do mundo no qual está inserido.

No processo de educação dos profissionais da saúde, as iniciativas de capacitação contínua têm sido caracterizadas pela relação com o processo de trabalho institucional, objetivando a transformação da prática. Para tanto, vem adotando como pressuposto pedagógico a discussão da realidade a partir dos elementos que façam sentido para os profissionais responsáveis pela busca na melhoria das condições de trabalho e da qualidade dos serviços. Neste processo de educação, a utilização de uma tecnologia de comunicação de ponta deve estar sustentada numa concepção de ensino que possibilite uma aprendizagem significativa, apoiada no pensamento reflexivo, dialógico, contextual, colaborativo e construtivo.

Corroborando com este cenário, o desenvolvimento científico e tecnológico na sociedade atual vem causando transformações constantes nos ambientes de trabalho e conseqüentemente, exigindo um profissional com perfil mais aberto e capaz de adaptar-se a mudanças, instrumentalizado e motivado a continuar aprendendo ao longo de sua vida.

$\mathrm{O}$ uso da tecnologia como ferramenta mediatizadora desses processos educacionais tem sustentado as iniciativas de capacitação, em especial aquelas de educação à distância, apresentando-se como mais uma alternativa de atualização profissional. Diante do exposto, objetiva-se contribuir com uma reflexão sobre perspectivas e desafios da educação em saúde e na enfermagem, tendo como ênfase o processo de capacitação profissional em serviço.

\section{Incorporação tecnológica: mudanças organizacionais e na educação}

As mudanças no mundo contemporâneo têm gerado transformações radicais na forma de produção e de relação entre os indivíduos. Nas últimas décadas, a revolução provocada 
pela informática nos ambientes empresariais e até mesmo domésticos, tem feito com que grande parte das pessoas altere seus hábitos. Tomando como base os períodos históricos da evolução da sociedade, marcados inicialmente pela produção manufatureira e, posteriormente, demarcados pela produção industrial e pós-industrial, constata-se que estes foram fortemente influenciados pela incorporação tecnológica nos processos de produção e que causaram grandes mudanças na forma de vida das pessoas.

Atualmente as conseqüências do ritmo imposto pela tecnologia, não têm acompanhado as transformações sociais, econômicas, políticas e espirituais dos indivíduos. Concordamos com Bell (1) quando enfatiza a perda de valores espirituais para explicar a crise contemporânea. $\mathrm{O}$ homem moderno tornou-se dependente da tecnologia para agir tanto no ambiente de trabalho como nas suas relações pessoais.

As transformações organizacionais não acontecem de forma linear e rápida, é necessário haver alterações em todos os processos internos de produção e mais ainda, uma interação entre os interesses e compromissos, tanto no âmbito individual, quanto no coletivo (dos grupos) dentro da organização. Dessa forma, pode-se dizer que a mudança na organização é um processo de aprendizagem, onde valores e ideais são questionados em busca de uma (re)definição política e ideológica e, conseqüentemente, de um novo desenho organizacional.

As mudanças ocorrem a partir do momento em que a flexibilização no modo de "fazer" o trabalho resultar em capacitação, adequação, incorporação e inovação importantes para a busca do cumprimento das diretrizes institucionais.

No entanto, a forma como as organizações prestadoras de serviço foram tradicionalmente estruturadas, a exemplo, as instituições de saúde, resulta em organizações complexas, hierarquizadas, com vários níveis de decisão e, portanto, pouco ágeis em re- lação ao processo de trabalho. Esse modelo de estrutura, ao longo dos anos, tem sido questionado em relação à produtividade, competitividade e atendimento às necessidades dos clientes impostas pelo mercado. Em conseqüência, novos modelos gerenciais são adotados, buscando definir um desenho que permita a introdução crescente de tecnologias de processo e de produto.

Em estudo realizado por Guimarães (2), na análise dos processos de mudança organizacional, fica evidente que o contexto da instituição, compreendido pelo ambiente interno e externo onde se insere, é de extrema importância. Por várias oportunidades, tem-se deparado com programas de mudança institucional que tentam implantar novos arranjos organizacionais sem, contudo, considerarem a transposição contextual, entendida na sua dimensão cultural, econômica, política e social, necessárias à adequação do modelo.

No contexto das mudanças organizacionais, Motta (3) chama a atenção para o termo desenvolvimento organizacional definido por Warren G. Bennis, que não se limita à mudança organizacional, mas é adotado como uma estratégia educacional que promove uma mudança planejada, enfatizando o comportamento baseado em experiências vividas na organização.

As transformações na sociedade atual desencadeadas pela globalização, avanços tecnológicos e, conseqüentemente, nas instituições, têm demandado também mudanças na área da educação, gerando novas maneiras de viver, de educar, de aprender e de se relacionar.

Segundo Nascimento e Prado (4) a tecnologia é entendida como uma arte, um conhecimento dos instrumentos, que depende da elaboração, da criação e da utilização apropriada, enquanto a técnica corresponde unicamente à maneira de se servir destes instrumentos.

Os profissionais de enfermagem em meio a estes avanços tecnológicos, permeados 
por uma variedade de instrumentos e técnicas, têm realizado esforços no sentido de incorporar a tecnologia no seu processo de trabalho como ferramenta para a mudança nos ambientes, sem, no entanto, considerá-la recurso primordial. Esta mudança requer do profissional uma constante atualização, visão crítica e reflexiva do trabalho, concomitante à adequação das tecnologias à realidade vivida, em benefício da qualidade do cuidado.

Nos ambientes de formação profissional, associado à incorporação tecnológica é imprescindível um agir comunicativo, baseado em entendimento mútuo, para que ocorra uma aproximação do docente e aluno, estimulando-os a descobertas, que submetidas ao processo de reflexão e análise, geram conhecimentos para a enfermagem, numa forma integrada entre o pensar e o fazer, resultando não apenas em ação ou procedimento técnico, mas em inovação.

Neste contexto, a educação deve ser pensada como um exercício coletivo de valorização das experiências e da criatividade individual, buscando novos instrumentos para o trabalho. Como conseqüência, educar torna-se o (re)inventar e a (re)construção do conhecimento de forma personalizada, transpondo o mero preparo de mão de obra para o mundo globalizado, buscando a capacitação do profissional, um transformador da realidade, um (re)avaliador crítico. Esta condição favorece uma (re)descoberta do indivíduo, das suas potencialidades, dos seus limites e de alguma maneira, subsidia o desenvolvimento de suas capacidades.

$\mathrm{Na}$ visão de Gadotti (5) "o traço mais original da educação deste século é o deslocamento do enfoque individual para o social, para o político e para o ideológico". Em outros estudos Gadotti e Romão (6) reforçam que pela educação deseja-se mudar o mundo, a começar pela sala de aula, pois as grandes transformações não se dão apenas como resultantes dos grandes gestos, mas de iniciativas cotidianas, simples e persistentes, num processo dialético entre o projeto pessoal e o coletivo.

No cenário da Enfermagem, o momento é de construção e vivência de um novo modelo pedagógico, enfatizando o desenvolvimento científico-tecnológico, que traz à tona questões relativas às transformações sociais, novos padrões de comportamento, alterando a forma de organização do trabalho. Iniciativas têm sido feitas no sentido de incentivar a utilização das tecnologias como instrumentos pedagógicos, envolvendo o serviço e a comunidade. No âmbito acadêmico, propõe o envolvimento de docentes e discentes com a realidade em que o trabalho ocorre, aliado a uma construção do conhecimento, extrapolando os limites da escola e revelando uma nova visão de ensino-aprendizagem (4).

Para estes autores, a construção do conhecimento deve ser permeada pelo diálogo e pela valorização das relações interpessoais, na busca pelo entendimento, não se realizando apenas com a explanação de idéias descontextualizadas da realidade, mas sim, com a mudança de atitude docente, e também dos alunos na vivência das relações sociais, e intercâmbio de experiências que possibilitem novas formas de pensar o saber e o fazer.

Ressalta-se que independente da modalidade utilizada, alguns aspectos são relevantes, tais como, engajar os alunos de forma dinâmica no processo de aprendizagem; estabelecer elos entre o aprendizado e a experiência dos alunos; fornecer aos alunos uma variedade de modalidades de aprendizagem; envolvimento do corpo docente.

Todos esses processos de mudanças fazem com que sejam questionadas as modalidades convencionais de educação, que não conseguem atender a crescente necessidade de formação de recursos humanos e de sua educação permanente, bem como as formas tradicionais de ensino, centradas no espaço do território da escola e da utilização de metodologias de educação centrada no professor.

Frente a este quadro, surge a necessidade de flexibilização de tempo, lugar, conteúdo 
de aprendizagem, recursos instrucionais, de modo a atender o aluno em suas necessidades e graus de liberdade individual.

\section{Educação em enfermagem: situação atual e perspectivas}

As demandas e necessidades que orientam as mudanças na formação do pessoal de enfermagem são determinadas pelas transformações no mundo do trabalho, pelas crescentes demandas por uma melhor atenção à saúde, pela vigilância à saúde e pelas críticas ao modelo de ensino tradicional, sustentado nas teorias centradas em conteúdos e objetivos pedagógicos.

Vivencia-se, no contexto da educação em enfermagem, o movimento de implantação das Diretrizes Curriculares (DC), que representam uma estratégia fundamental para atender as necessidades e demandas na formação dos profissionais de enfermagem. As DC constituem-se em um instrumento norteador do processo de construção dos projetos políticos pedagógicos das escolas e devem fornecer as bases filosóficas, conceituais, políticas e metodológicas do processo de formação dos enfermeiros, além de fornecer o apoio financeiro.

Segundo Silva e Sena (7) implantar diretrizes implica em promover mudanças no âmbito educacional, que passa a exigir da escola a criação de espaços e mecanismos para a reflexão e tomada de decisão coletiva. Estes espaços devem ser detonadores de dispositivos capazes de mover docentes, estudantes e profissionais do serviço para novas possibilidades. O movimento de transformação requer, ademais, a disponibilidade de tecnologias, uma decisão política e institucional e o estabelecimento de estratégias para a construção das mudanças, que devem ter como intencionalidade, novos modos de aprender, de assistir e de cuidar.

A adoção deste novo paradigma educacional envolve resistências e conflitos, devido ao confronto com distintas e, às vezes, contradi- tórias concepções, e à resistência que emerge do dilema de um posicionamento diante de uma ou outra concepção, ou da construção de uma própria.

Esta realidade pode ser percebida tanto no corpo docente quanto discente. A resistência para mudança do paradigma educacional é percebida na resistência por parte de docentes em incorporar concepções pedagógicas que estimulem o desenvolvimento da consciência crítica e a participação ativa do aluno; na escassa variedade de métodos e materiais educativos (desconhecimento, dificuldade de utilização e alto custo); na dificuldade de aceitação, por parte dos estudantes, da responsabilidade pela auto-aprendizagem por meio da participação ativa na produção do conhecimento e, ainda, na rigidez das instituições formadoras de recursos humanos, no que se refere às questões técnicas e administrativas, dificultando a implementação de propostas inovadoras.

Com relação especificamente aos docentes, podem ser acrescidas algumas limitações para o uso de metodologias inovadoras no processo de educação, tais como, o medo de perder conteúdos do seu domínio, medo do novo, medo de perder espaços nas disciplinas, dificuldade de trabalhar com metodologias ativas de ensino e implementar a interdisciplinaridade na construção da articulação do conhecimento.

Para alguns autores (8) a implementação de metodologias inovadoras deve ser aplicada desde que a instituição educacional flexibilize o planejamento pedagógico-didático, de tal forma que docentes de diferentes disciplinas possam discutir e construir atividades de ensino interligadas, com disponibilização de horários e espaços físicos, a fim de viabilizar as atividades planejadas e propostas em conjunto. Nesta proposta, os autores caracterizam o professor como dodiscente e o estudante como um didoscente, ou seja, professores e alunos estão continuamente articulados na relação de ensino e aprendizado, considerando ser este um processo inacaba- 
do e inconcluso, no qual o grupo estabelece as grandes temáticas a serem tratadas.

Entende-se, portanto, que o processo educativo vai além da produção do conhecimento, sendo indispensável "a socialização da produção, necessária para a transformação social por meio da democratização efetiva, com a concretização de um projeto político pedagógico em permanente construção, com foco no ensino, pesquisa e a extensão, como finalidade da educação"(9).

\section{Educação Permanente em Saúde e na Enfermagem: novos desafios}

Apesar de muito se discutir sobre educação em serviço, o seu papel na reorganização dos modelos assistenciais e no processo de trabalho ainda é pouco impactante, pois na maioria das vezes, não considera a realidade da instituição e a experiência do trabalhador. Muitas são as denominações empregadas a esse tipo de educação, as que aparecem com maior freqüência são: educação contínua ou continuada, educação em serviço e educação permanente.

A expressão educação permanente conforme citado por Coleet (10) foi utilizada oficialmente em 1960, no Congresso Mundial de Educação de Adultos, realizado pela UNESCO na cidade de Montreal, Canadá. Este conceito foi adotado por países subdesenvolvidos e, no Brasil, por volta de 1970. Ribeiro e Motta (11) citam que a educação continuada aparece pela primeira vez de forma explícita durante a VI Conferência Nacional de Saúde em 1977.

De acordo com Martins (12), a educação do mundo atual deve romper com os modelos tradicionais de educação do passado, de modo que o sistema escolar não mais projete a educação sem ter como referência a educação permanente, da mesma forma, que esta última não poderá ignorar a realidade escolar e tão pouco desobrigar-se de uma análise de sua dimensão institucional.

Para Ceccim (13) o conceito de Educação
Permanente em Saúde é ampliado considerando que a mesma corresponde à Educação em Serviço, e também quando se alia a Educação Continuada e a Educação Formal de Profissionais. Dessa forma,

[...] a educação permanente corresponde a educação em serviço quando esta coloca a pertinência dos conteúdos, instrumentos e recursos para a formação técnica, submetidos a um projeto de mudanças institucionais ou de mudanças da orientação política, das ações prestadas em um dado tempo e lugar. Corresponde à educação continuada quando esta pertence a construção objetiva de quadros institucionais e a investidura de carreiras por serviços em tempo e lugar. Por fim, corresponde à educação formal de profissionais quando esta se apresenta amplamente porosa às multiplicidades da realidade de vivências profissionais e coloca-se em aliança de projetos integrados entre o setor mundo do trabalho e o setor mundo de ensino (13).

A Educação Permanente ganhou estatuto de política pública na área da saúde, devido à difusão pela Organização Pan-Americana de Saúde (OPAS), da proposta de Educação Permanente do Pessoal de Saúde para alcançar o desenvolvimento dos sistemas de saúde na região, considerando que os serviços de saúde são organizações complexas, em que apenas o aprendizado significativo será capaz de gerar a adesão dos trabalhadores aos processos de transformação do cotidiano (13).

Após o Ministério da Saúde ter assumido o papel de formulador de políticas orientadas para a formação de recursos humanos em saúde, vários foram os programas implementados na tentativa de aprimorar a atenção à saúde da população. Apesar destas iniciativas terem possibilitado a aproximação de profissionais dos serviços de saúde e das instituições formadoras, as mudanças alcançadas foram pontuais, não resultando em modificações profundas almejadas.

Em 2004, o Ministério da Saúde (14) pro- 
põe uma nova Política de Educação e Desenvolvimento para o SUS: Caminhos para a Educação Permanente em Saúde, alimentando a articulação por meio de três eixos fundamentais: a relação entre educação e trabalho, mudança na formação e a produção de conhecimento.

Nesta política, a Educação Permanente utilizada como estratégia fundamental para recomposição das práticas de atenção, de gestão e do controle social no setor da saúde, é definida como a concretização do encontro entre o mundo da formação e o mundo de trabalho, onde o aprender e o ensinar se congregam ao cotidiano das organizações e ao trabalho, ou seja, a educação permanente trabalha com as transformações das práticas técnicas e sociais, partindo sempre da realidade em que os sujeitos envolvidos estão inseridos.

A educação permanente em saúde é definida ainda, como uma

[...] estratégia dinâmica e eficaz para gerar novos modelos e processos de trabalho nos estabelecimentos de saúde. Tem como objetivo a reflexão e intervenção nos processos de trabalho, na melhoria da qualidade dos serviços e nas condições de trabalho, sendo sintetizada pela OPAS como a educação em trabalho, para o trabalho e pelo trabalho (15).

O modelo pedagógico de escolha para as iniciativas de educação permanente em saúde -análise crítica da prática com a problematização- permite capacitar todos os profissionais de um mesmo serviço, devendo ser uma tarefa permanente, sustentada pela participação multidisciplinar dos trabalhadores. É ainda, uma ferramenta valiosa para o diagnóstico das necessidades de capacitação dos profissionais da saúde, pois como tal, permite explorar as necessidades do ponto de vista dos trabalhadores, em função dos problemas identificados em sua prática diária. Demo (16) corroborando com esta afirmativa, cita que o processo de aprendizagem é perma- nente, não só por que nunca é viável atingir um ponto final, mas porque significa movimento de recriação infinita.

Neste sentido, implementar os processos de educação permanente em saúde implica em trabalhar com uma estratégia que inclua o desenvolvimento de processos de mudança, em especial, na prática do serviço de saúde e na instituição como um todo. Por conseguinte, a estratégia de educação permanente em saúde permite alcançar o desenvolvimento simultâneo dos recursos humanos e do serviço, quer dizer, do trabalhador e do trabalho, uma vez que a melhoria das competências organizacionais e prestações de serviços dos profissionais, permitem melhorar a qualidade da atenção, garantindo, portanto, a maior satisfação dos usuários do serviço de saúde. Assim, mais que as legítimas aspirações individuais, educação permanente em saúde é destinada basicamente a satisfazer as necessidades dos processos de trabalho, de prestação, de organização e de produção de serviços que são a razão de ser dos estabelecimentos de saúde.

Para a concretização dos processos de educação permanente são requeridos métodos e técnicas que avançam no sentido da adoção de uma atitude facilitadora por parte do tutor e na co-responsabilidade do aluno na produção do conhecimento, facilitadas pelo uso de tecnologias como mediadoras do processo de educação, tal qual, empregadas nas experiências de educação à distância.

Neste contexto, Belloni (17) destaca que a interação das novas tecnologias de informação e comunicação nos processos educacionais pode ser uma estratégia de grande valia, desde que considere estas técnicas como meios e não como finalidades educacionais, e que elas sejam, ao mesmo tempo, utilizadas como ferramentas pedagógicas e como objeto de estudo complexo e multifacetado com uma abordagem crítica e criativa das mesmas.

Esta premissa é também confirmada por Peres e Kurcgant (18) quando reconhecem 
que as novas tecnologias aplicadas ao ensino (Internet, robótica e realidade virtual) permitem maior flexibilidade, criatividade, dinamicidade, interação e comunicação no processo ensino e aprendizagem, resultando na participação ativa do aluno na construção do conhecimento.

Entretanto, Moran (19) salienta que não podemos ver a Internet como solução mágica para modificar a relação pedagógica. Nestas experiências, o professor deve valorizar o diálogo, a troca, a relação interpessoal, rompendo com o modelo de ensino conservador e tradicional. Ensinar e aprender à distancia são atividades que demandam dedicação e responsabilidade, desafiando aluno e professor a modificarem seus papéis, o aluno deve ter mais iniciativa, motivação e disciplina, enquanto o professor deve trabalhar como consultor, colaborador e facilitador do aprendizado. Além disto, é fundamental ressaltar a importância da interação, do diálogo e do intercâmbio de idéias e experiências entre os tutores, profissionais de saúde e os participantes do processo de educação para a construção do conhecimento.

Nesta nova perspectiva de aprendizagem, Demo (16) enfatiza que é necessária a presença crítica e criativa do sujeito para que ocorra a aprendizagem, por que releva o sentido da conquista da autonomia por fazer ligação direta entre o saber pensar e saber aprender. Desta forma, "aprender é, no seu âmago, saber fazer-se sujeito de história própria, individual e coletiva" (16).

Para que a educação ocorra com êxito é imprescindível, além de programas e sistemas de qualidade para desenvolvimento das atividades de educação, recursos humanos capacitados, material didático adequado e centros de apoio para orientação do corpo docente e discente.

A educação à distância pode ser utilizada tanto como forma complementar de educação como forma substituta da educação presencial, mas sempre com a vantagem de permitir uma participação coletiva, com produções coletivas mesmo que aconteça em lugares distintos, com horários distintos, ou seja, uma comunicação assíncrona, favorecida pela velocidade da comunicação e menor custo.

Pelo exposto, o desenvolvimento de programas de educação deve ser orientado pela evolução da tecnologia que demanda rápidas mudanças no contexto do cuidado à saúde, devendo também ser organizado de forma a trabalhar as necessidades educativas identificadas em cada grupo. É de reconhecimento amplo que a educação permanente é de grande valor, pois proporciona conhecimento, crescimento, atualização e aperfeiçoamento do profissional.

No entanto, tem que se questionar ainda, se o conhecimento atualizado é suficiente para modificar os processos, o trabalho e as relações entre os profissionais, isto é, qual o verdadeiro reflexo da educação nas práticas profissionais. Com esta reflexão procura-se apenas iniciar um debate sobre as perspectivas da educação, sem intenção de encerrá-la, cientes de que são algumas pistas e que existem muitos outros desafios a serem enfrentados. Considera-se, portanto, que tão importante quanto a reflexão critica, é a prática sem a reflexão sobre ela, ou seja, uma análise em profundidade por aqueles envolvidos com o tema.

\section{REFERÊNCIAS}

1. Bell D. The coming of post-industrial society. New York: Basic Books, 1973.

2. Guimarães EMP. Sistema de Informação Hospitalar: Instrumento para tomada de decisão no exercício da gerencia de unidades funcionais [tese doutorado]. São Paulo: Escola de Enfermagem de Ribeirão Preto da Universidade de São Paulo; 2004. 
3. Motta FCP. Teoria das organizações: evolução e crítica. São Paulo: Pioneira; 1995.

4. Nascimento SR, Prado ML. O agir comunicativo na construção do conhecimento em enfermagem. Rev. bras. enferm. 2004; 57(2):237-40.

5. Gadotti M. Perspectivas atuais da educação. São Paulo em Perspectiva. 2000; 14 (02): 3-11.

6. Gadotti M, Romão JE. Educação de jovens e adultos - teoria, prática e propostas. São Paulo: Cortez; 2000.

7. Silva KL, Sena RR. O processo de mudança na educação de enfermagem nos cenários UNI: potencialidades e desafios. Rev. bras. enferm. 2003; 56(4):412-6.

8. Silva CRLD, Keim EJ, Bertoncini JH. Transdisciplinaridade na educação para a saúde: um planejamento para a graduação do enfermeiro. Rev. bras. enferm. 2003; 56(4):424-8.

9. Dias DC, Murrofuse NT, Schneider JF, Tonini NS, Oliveira BRG. Reflexões sobre o desafio da administração da educação em enfermagem. Rev. bras. enferm. 2004; 57(4):490-2.

10. Vilarinho LR. Educação continuada e educação a distância: anomalias no contexto do paradigma tradicional de ensino? 24a Reunião Anual da Associação Nacional de Pós-Graduação e Pesquisa em Educação (ANPED) 2001. Hallado em:http://www.anped.org.br/reunioes/24/T0442890705758.DOC. [Acesso em 07/01/05].

11. Ribeiro ECO, Motta JIJ. A educação permanente como estratégia na reorganização dos serviços de saúde. 2005. Hallado em: http://www.redeunida.org. br/producao/artigo03.asp. [Acesso em 29/07/2005].
12. Martins OB. A Educação Superior a distância, uma modalidade de educação permanente para a UFPR. Rev Educação a Distância, 1994; (4-5). Hallado em: http://www.intelecto.net/ead_textos / onilza1.html. [Acesso em: 10/01/2005].

13. Ceccim RB. Educação Permanente em Saúde: desafio ambicioso e necessário. Interface (Botucatu). 2005; 9 (6): 161168. Hallado em: http://www.interface. org.br/revista16/debate1.pdf. [Acesso em 03/01/2005].

14. Ministério da Saúde. Brasil. Política de Educação e Desenvolvimento para o SUS. Caminhos para a Educação Permanente em Saúde: Pólos de Educação Permanente em Saúde. Brasília; 2004.

15. Miranda E. El análisis crítico de la práctica en el marco de la educación permanente del personal de salud: orientaciones metodológicas para su aplicación. Lima: MINSA; 2002. Pp. 47.

16. Demo P. Saber pensar. 4a ed. São Paulo: Cortez, Instituto Paulo Freire; 2005. Pp.51.

17. Belloni ML. Ensaio sobre a educação a distância no Brasil. Educ. Soc. 2002; 23(78): 117- 142. Hallado em: http://www.scielo.br/ scielo.php?script=sci_arttext\&pid=S010173302002000200008\&lng =es\&nrm=isso. [Acesso em 14/01/05].

18. Peres HHC, Kurcgant P. O ser docente de enfermagem frente a Informática. Rev. Latino-Am. Enfermagem. 2004; 12(1):101108. Hallado em: http://www.scielo.br/ scielo.php?script=sci_arttext\&pid=S0104$11692004000100014 \& \operatorname{lng}=\mathrm{pt} \& \mathrm{nrm}=$ isso. [Acesso em 11/01/2005].

19. Moran JM. Novos desafios na educação-a Internet na educação presencial e virtual 2001. Hallado em: http://www.eca.usp. br/prof/moran/novos.htm. [Acesso em 12/01/05]. 